\title{
Post-stent management with a pneumatic groin compression device and self injected low molecular weight heparin
}

\author{
R H Stables, U Sigwart
}

\begin{abstract}
Background-The benefits of intracoronary stent implantation are offset by an increased risk of complications at the arterial puncture site and a prolonged hospital stay. Much of this morbidity can be attributed to the generally perceived need to achieve systemic anticoagulation after stent implantation.

Aim-To test a simplified protocol for post-stent management using the Femostop pneumatic groin compression device and low molecular weight (fractionated) heparin (LMWH) administered by subcutaneous injection.

Patients-A case series of 100 consecutive patients, with stable angina pectoris, undergoing coronary stenting for a suboptimal result after conventional balloon angioplasty.

Methods-All patients were managed with a new post-stent protocol using the Femostop pneumatic groin compression device and LMWH. The incidence of complications and the length of hospital stay were recorded.

Results-The clinical course was uncomplicated in 92 patients and their discharge from hospital was achieved on the first post-procedural day for 44 patients and on the second for the remaining 48 . The rate of vascular or bleeding complications was $6 \%$.

Conclusions-LMWH administered by subcutaneous injection may provide a practical and effective alternative to the use of intravenous heparin when systemic anticoagulation is used after stent implantation.
\end{abstract}

(Heart 1996;75:588-590)

Keywords: stent; low molecular weight heparins; anticoagulation; compression device

Department of Invasive Cardiology, The Royal Brompton Hospital, London R H Stables U Sigwart

Correspondence to: Dr U Sigwart, Department of Invasive Cardiology, The Royal Brompton Hospital, Sydney Street, London SW3 6NP.

Accepted for publication 17 October 1995
The benefits of intracoronary stent implantation are offset by an increased risk of complications at the arterial puncture site and a prolonged hospital stay. ${ }^{12}$ Much of this morbidity can be attributed to the generally perceived need to induce, monitor, and maintain systemic anticoagulation after stent implantation. We have tested a simplified protocol for post-stent management using the Femostop pneumatic groin compression device and low molecular weight (fractionated) heparin
(LMWH) administered by subcutaneous injection.

\section{Patients and methods}

A case series of 100 consecutive patients with stent implantation by the senior author (US) is described. All patients underwent elective percutaneous transluminal coronary angioplasty (PTCA) for the treatment of stable angina. The indication for stenting was a suboptimal result after balloon dilatation. A total of 123 stents (PS-153, Johnson and Johnson Interventional Systems) were implanted in the native coronary circulation $(n=74)$ and in saphenous vein grafts $(n=26)$. Tandem deployment of two stents was used in 12 patients and a half stent was implanted in a further 24 .

Intravascular ultrasound imaging was used in only 10 patients but high pressure balloon inflation (15 (3) atm) was uniformly applied. The mean (SD) diameter of the reference vessel was $3.24(0.27) \mathrm{mm}$ and the mean (SD) final balloon diameter was $3.4(0.6) \mathrm{mm}$.

We elected to maintain systemic anticoagulation after implantation. Aspirin at a dose of $300 \mathrm{mg}$ daily and a calcium antagonist were prescribed for all patients scheduled for a coronary intervention. Warfarin was administered, as a loading dose of $15 \mathrm{mg}$, on the day of $(n=68)$ or the day before $(n=32)$ the procedure and continued for 6 weeks. The dose was adjusted to maintain an international normalised ratio (INR) in the range 2.5-3.5.

Intravenous heparin was administered as a bolus injection of 15000 units at the start of the PTCA. The mean (SD) activated clotting time (ACT) at implantation was $346(48) \mathrm{s}$. On return to the ward the ACT was tested and the arterial sheath removed when the ACT value fell below $180 \mathrm{~s}$. Groin compression was applied using a Femostop groin compression system. The inflation pressure of the pneumatic dome was adjusted to the systolic arterial pressure for some $20-30 \mathrm{~min}$ to secure initial haemostasis. Groin compression was then continued at low pressures $(20-40 \mathrm{~mm} \mathrm{Hg}$ ) for an additional 6-8 $\mathrm{h}$ and mobilisation then encouraged.

Treatment with LMWH (dalteparin sodium-Fragmin, Pharmacia, Milton Keynes, UK) was initiated on the following day and administered at a dose of 5000 units twice daily by subcutaneous injection. Patients were instructed in the self administration of this agent and use was continued for 7 days or 
until a therapeutic INR result was obtained. Outpatient estimation of the INR and warfarin dose adjustment were arranged with the patient's primary or secondary care physicians.

\section{Results}

The clinical course was uncomplicated in 92 patients and their discharge from hospital was achieved on the first post-procedural day for 44 patients and on the second for the remaining 48.

One patient required immediate coronary artery bypass grafting for abrupt vessel closure. There was one subacute thrombosis $16 \mathrm{~h}$ after implantation, which was successfully treated with thrombolysis. This event was probably related to incomplete coverage of a distal dissection. One patient died at 10 days of haemorrhage from a dissection of the iliac artery presumably related to guidewire or catheter trauma at the time of the implantation procedure. One patient with a false aneurysm responded to compression treatment. A groin haematoma greater than $6 \mathrm{~cm}$ in diameter developed in four patients. The overall rate of vascular or bleeding complications in this series was thus $6 \%$; no transfusion or other intervention was required in two thirds of these.

\section{Discussion}

Bleeding and other peripheral vascular complications currently limit the value of coronary stenting. In the BENESTENT trial ${ }^{1}$ patients selected for a coronary intervention were randomised to stent implantation or PTCA. Puncture site complications were observed in $2.4 \%$ of patients undergoing PTCA and in $14.6 \%$ of those receiving a stent. The mean duration of hospital stay was prolonged from 3 days after PTCA to 9 days after stent implantation. Much of this morbidity can be related to the intensive anticoagulation regimen demanded by the trial protocol and several initiatives have attempted to address these issues.

Pneumatic groin compression devices have proven safe and effective and their use was associated with a 10-fold reduction in bleeding and other complications at one American centre. ${ }^{3}$ Other operators have explored alternative vascular access sites and good results have been reported from a brachial ${ }^{4}$ or radial $^{5}$ approach.

Most vascular complications occur in the immediate post-procedural phase after removal of the arterial sheath. Although warfarin treatment may have been initiated, systemic anticoagulation is usually secured in this period with an intravenous infusion of heparin. The administration rate is conventionally adjusted to maintain the kaolin cephalin clotting time, or similar test, at a value of two to three times greater than control and the infusion continued until blood tests demonstrate warfarin effect, with an INR in the target range. This approach necessitates inpatient care and repeated blood sample analysis, placing considerable demands on medical, nursing, and laboratory resources. We elected to use a LMWH preparation administered by subcutaneous injection to provide anticoagulation over this period. Patients undergoing stent implantation for the management of an acute coronary syndrome were excluded from this series and the safety and efficacy of the regimen in this group cannot be assumed.

LMWH preparations are mixtures of fractionated heparin molecules with sizes in the range $3000-10000 \mathrm{Da}$. The products of different manufacturers may exhibit different pharmacological and clinical properties and should be regarded as separate agents. ${ }^{6}$ All preparations, however, share important advantages over conventional, unfractionated heparin. Principal among these is a greater therapeutic index (less risk of bleeding for a given antithrombotic effect) and apparently no requirement for anticoagulant monitoring. ${ }^{7}$ Indeed, routine laboratory tests do not reflect their anticoagulant action and special assays of factor Xa activity are required.

They have greater biological availability after subcutaneous administration and a longer half life, allowing a dose regimen based on one or two daily injections. More recently, it has been reported that heparin induced thrombocytopenia (and its attendant prothrombotic state) is less common in patients treated with LMWH rather than unfractionated heparin. ${ }^{8}$

The mode of action of LMWH preparations is not fully characterised. They inhibit the activity of factor $\mathrm{Xa}$ but inhibition of thrombin formation and local effects at the endothelial level may also be important. ${ }^{9}$ They are of proven value in post-surgical thromboprophylaxis ${ }^{10}$ and in the treatment of deep venous thrombosis ${ }^{112}$ but their role and efficacy in other clinical situations are yet to be determined.

After stent implantation, they appear to provide a safe alternative to intravenous heparin, with easy administration by subcutaneous injection and no requirements for anticoagulant monitoring or dose titration. If patients can be instructed to self inject then hospital discharge can be secured on the first or second postoperative day. The responsibilities for warfarin dose adjustment are then devolved to physicians involved in shared care and their cooperation is essential to the success of this approach.

It is now recognised that, while bleeding complications are closely related to the intensity and duration of anticoagulation treatment, stent thrombosis is influenced by various factors, particularly the geometry of stent deployment and subsequent flow characteristics. ${ }^{13}$ Advances in stent design and implantation techniques have allowed a move to less intensive anticoagulation regimens. Some operators have now abandoned the use of postprocedural systemic anticoagulation, prescribing the antiplatelet agents aspirin and ticlopidine, either singly or in combination, for antithrombotic prophylaxis. ${ }^{14}$ Notwithstanding these developments, most operators still 
employ systemic anticoagulation after stent implantation and a management protocol using $\mathrm{LMWH}$ in the immediate post-procedural phase may provide a practical and effective alternative. Comparative trials with a prospective, randomised, controlled design are required to fully assess the value of these new management initiatives.

1 Serruys PW, De Jaegere P, Kiemeneij F, et al. A comparison of balloon-expandable-stent implantation with balloon angioplasty in patients with coronary artery disease. $N$ Engl $\mathscr{f}$ Med 1994;331:489-95.

2 Fischman DL, Leon MB, Baim DS, et al. A randomised comparison of coronary-stent placement and balloon angioplasty in the treatment of coronary artery disease. $N$ Engl $\mathcal{Y}$ Med 1994;331:496-501.

3 Sridhar K, Porter D, Gupta B, et al. Reduction in peripheral vascular complications after coronary stenting by the use of a pneumatic vascular compression device. Circulation 1994;90:1621.

4 Batin PD, Nolan J, Welsh CJ, McLenachan JM, Perrins EJ. Should all elective coronary stents be implanted via the brachial approach? Audit of a single centre experience of stenting from the femoral and brachial routes [abstract]. Br Heart f 1995; 73:P86.

5 Kiemeneij F, Laarman GJ, Slagboom T, Stella P.
Transradial Palmaz Schatz coronary stenting on an outpatient basis: results of a prospective pilot study. Fournal of Invasive Cardiology 1995;7:5-11A.

6 Wolf H. Low-molecular-weight heparin. Med Clin North Am 1994;78:733-43.

7 Hirsh J. Rationale for development of low-molecularweight heparins and their clinical potential in the preven1991;161:512-8.

8 Warkentin TE, Levine MN, Hirsh J, et al. Heparin induced thrombocytopenia in patients treated with lowmolecular-weight heparin or unfractionated heparin. $N$ Engl f Med 1995;332:1330-5.

9 Samama MM, Bara L, Gerotziafas GT. Mechanisms for the antithrombotic activity in man of low molecular the antithrombotic activity in man of low molecular weight

10 Jorgensen LN, Wille-Jorgensen P, Hauch O. Prophylaxis of postoperative thromboembolism with low molecular weight heparins. Br ₹ Surg 1993;80:689-704.

11 Hull RD, Pineo GF. Low molecular weight heparin treatment of venous thromboembolism. Prog Cardiovasc Dis 1994;37:71-8.

12 Cziraky MJ, Spinler SA. Low-molecular-weight heparin for the treatment of deep-vein thrombosis. Clin Pharm 1993;12:892-9.

13 Colombo A, Hall P, Nakamura S. Intracoronary stenting without anticoagulation accomplished with intravascular ultrasound guidance. Circulation 1995;91:1676-88.

14 Morice M. Advances in post stenting medication protocol [abstract]. Fournal of Invasive Cardiology 1995;7:32. 